\title{
PRECONDITIONING FOR THE STEADY-STATE NAVIER-STOKES EQUATIONS WITH LOW VISCOSITY
}

\author{
HOWARD C. ELMAN* \\ Report CS-TR-3712 \\ UMIACS-TR-96-82
}

November 1996

\begin{abstract}
We introduce a preconditioner for the linearized Navier-Stokes equations that is effective when either the discretization mesh size or the viscosity approaches zero. For constant coefficient problems with periodic boundary conditions, we show that the preconditioning yields a system with a single eigenvalue equal to one, so that performance is independent of both viscosity and mesh size. For other boundary conditions, we demonstrate empirically that convergence depends only mildly on these parameters and we give a partial analysis of this phenomenon. We also show that some expensive subsidiary computations required by the new method can be replaced by inexpensive approximate versions of these tasks based on iteration, with virtually no degradation of performance.
\end{abstract}

${ }^{*}$ Department of Computer Science and Institute for Advanced Computer Studies, University of Maryland, College Park, MD 20742, e-mail: elman@cs.umd.edu. This work was supported by U. S. National Science Foundation under grant DMS-9423133. 


\title{
PRECONDITIONING FOR THE STEADY-STATE NAVIER-STOKES EQUATIONS WITH LOW VISCOSITY
}

\author{
HOWARD C. ELMAN*
}

\begin{abstract}
We introduce a preconditioner for the linearized Navier-Stokes equations that is effective when either the discretization mesh size or the viscosity approaches zero. For constant coefficient problems with periodic boundary conditions, we show that the preconditioning yields a system with a single eigenvalue equal to one, so that performance is independent of both viscosity and mesh size. For other boundary conditions, we demonstrate empirically that convergence depends only mildly on these parameters and we give a partial analysis of this phenomenon. We also show that some expensive subsidiary computations required by the new method can be replaced by inexpensive approximate versions of these tasks based on iteration, with virtually no degradation of performance.
\end{abstract}

Key words. Navier-Stokes, Oseen, preconditioning, iterative methods.

AMS(MOS) subject classifications. Primary: 65F10, 65N20; secondary 15 A06

1. Introduction. This paper introduces a methodology for preconditioning the discrete steady-state incompressible Navier-Stokes equations

$$
\begin{aligned}
-\nu \Delta \mathbf{u}+(\mathbf{u} \cdot \operatorname{grad}) \mathbf{u}+\operatorname{grad} p & =\mathbf{f} \\
-\operatorname{div} \mathbf{u} & =0
\end{aligned} \quad \text { in } \Omega,
$$

subject to suitable boundary conditions on $\partial \Omega$, where $\Omega$ is an open bounded domain in $\mathbb{R}^{2}$ or $\mathbb{R}^{3}$. The vector field $\mathbf{u}$ represents the velocity in $\Omega, p$ represents pressure, and the scalar $\nu$ is the viscosity, which is inversely proportional to the Reynolds number. We will develop the preconditioners for the linearized version of (1) known as the Oseen equations, which can be written as

$$
\begin{aligned}
-\nu \Delta \mathbf{u}+(\mathbf{w} \cdot \operatorname{grad}) \mathbf{u}+\operatorname{grad} p & =\mathbf{f} \\
-\operatorname{div} \mathbf{u} & =0
\end{aligned}
$$

where $\mathbf{w}$ is given such that $\operatorname{div} \mathbf{w}=0$. These equations arise from a nonlinear iteration essentially of the form $-\nu \Delta \mathbf{u}^{(m)}+\left(\mathbf{u}^{(m-1)} \cdot \operatorname{grad}\right) \mathbf{u}^{(m)}+\operatorname{grad} p^{(m)}=\mathbf{f},-\operatorname{div} \mathbf{u}^{(m)}=0$, see $[17]$.

Discretization of (2) yields a linear system of equations

$$
\left(\begin{array}{cc}
F & B^{T} \\
B & 0
\end{array}\right)\left(\begin{array}{l}
\mathbf{u} \\
p
\end{array}\right)=\left(\begin{array}{l}
\mathbf{f} \\
0
\end{array}\right),
$$

where $\mathbf{u}$ and $p$ now represent discrete versions of velocity and pressure, respectively. Here $F=\nu A+N$ where $A$ consists of a set of uncoupled discrete Laplace operators, corresponding to diffusion, and $N$ is a discrete convection operator. We are interested in convergence behavior of iterative methods applied to (3) as either $\nu$ or the discretization mesh width $h$ tend to zero. For small $\nu$ it is necessary for the discretization to be fine enough to resolve features such as boundary layers [13]; typically when $\nu$ is decreased, $h$ will also be reduced proportionally. We are concerned only with

\footnotetext{
* Department of Computer Science and Institute for Advanced Computer Studies, University of Maryland, College Park, MD 20742, e-mail: elman@cs.umd.edu. This work was supported by U. S. National Science Foundation under grant DMS-9423133.
} 
values of $\nu$ for which stable steady-state solutions exist; for example, it is shown in [12] that values of $1 / \nu$ on the order of 1000 to several thousand are feasible.

Let $\mathcal{A}$ denote the coefficient matrix of (3). We will consider preconditioners of the form

$$
\mathcal{Q}=\left(\begin{array}{cc}
F & B^{T} \\
0 & -X
\end{array}\right)
$$

It is easily confirmed that

$$
\mathcal{A} \mathcal{Q}^{-1}=\left(\begin{array}{cc}
I & 0 \\
B F^{-1} & B F^{-1} B^{T} X^{-1}
\end{array}\right)
$$

so that the eigenvalues of $\mathcal{A Q}^{-1}$ are

$$
\{1\} \cup \sigma\left(B F^{-1} B^{T} X^{-1}\right)
$$

Let $M$ denote the diagonal of the pressure mass matrix associated with the discretization; for finite differences, a natural analogue is $M=h^{d} I$ for a uniform grid of width $h$ in $d$ dimensions. It was shown in [5] that for the choice $X=\frac{1}{\nu} M$, the eigenvalues of $B F^{-1} B^{T} X^{-1}$ are bounded independent of $h$. (See $[2,22,25,29]$ for analogous results for the Stokes equations.) Therefore, use of this preconditioner in conjunction with Krylov subspace methods such as GMRES [24] leads to asymptotic convergence rates that are independent of the mesh size. Similar asymptotic bounds were also obtained in [11] for a different class of preconditioners based on the symmetric part of $\mathcal{A}$, where the preconditioning entails solution of the Stokes equations.

The convergence properties of these approaches depend on the viscosity $\nu$, and in general convergence rates deteriorate as $\nu$ decreases. For example, the results in [5] yield eigenvalues that are contained in a box in the complex plane of the form

$$
\left[c_{1} \nu^{2}, c_{2}\right] \times i\left[c_{3}, c_{4}\right]
$$

where $\left\{c_{j}\right\}$ are independent of $h$ and $\nu$, and in experimental results iteration counts increase roughly like $1 / \nu$. (See also $\$ 4$.)

Our concern here is to develop alternative choices for $X$ for which the sensitivity to $\nu$ is less pronounced. Our starting point is an observation derived from [6]. Let $G$ and $K$ be two matrices of dimensions $n_{p} \times n_{u}$ with $n_{u} \geq n_{p}$ and such that both are of full rank $n_{p}$. The matrix $K^{T}\left(G K^{T}\right)^{-1} G$ can then be viewed as an operator from range $\left(K^{T}\right)$ to itself, and it is trivial to see that this is in fact the identity operator. To apply this to (3), first assume for simplicity that $B$ is of full rank (this assumption will be eliminated below) and let $G=B F^{-1}$ and $K=B$. Our observation is then

$$
B^{T}\left(B F^{-1} B^{T}\right)^{-1} B F^{-1}=I \quad \text { on } \operatorname{range}\left(B^{T}\right)
$$

or, equivalently,

$$
B^{T}\left(B F^{-1} B^{T}\right)^{-1} B=F \quad \text { on } \operatorname{range}\left(F^{-1} B^{T}\right)
$$

Suppose for the moment that

$$
\operatorname{range}\left(B^{T}\right) \subset \operatorname{range}\left(F^{-1} B^{T}\right) .
$$


Then the equality of (6) can be postmultiplied by $B^{T}$, and premultiplying the result by $B$ yields

$$
\left(B B^{T}\right)\left(B F^{-1} B^{T}\right)^{-1}\left(B B^{T}\right)=B F B^{T} .
$$

Equivalently,

$$
\left(B F^{-1} B^{T}\right)^{-1}=\left(B B^{T}\right)^{-1}\left(B F B^{T}\right)\left(B B^{T}\right)^{-1} .
$$

That is, for the choice

$$
X=\left(B B^{T}\right)\left(B F B^{T}\right)^{-1}\left(B B^{T}\right)
$$

the eigenvalues of the preconditioned operator (5) are identically 1 and one step of GMRES will produce the solution.

In the rest of this paper, we examine the use of the preconditioner (4) for solving the discrete two-dimensional Oseen equations, using $X$ defined by (8) as well as some computationally less expensive variants. Because of the presence of the high-order discrete operator $B F B^{T}$ in (8), we will refer to the combination of (4) and (8) as the "BFBt preconditioner." Cf. [20] for other approaches to the problem of approximating the action of the inverse of $B F^{-1} B^{T}$. For our analysis, we restrict our attention to a "marker-and-cell" (MAC) finite-difference operator [15], which we outline in $\S 2$. In $\S 3$, we show that in the case where (2) is given with constant "wind" w and periodic boundary conditions, ( 7 ) holds. (In fact the two spaces are identical.) Consequently, in this case the discussion of the previous paragraph represents a complete analysis. In $\S 4$, we examine through a series of numerical experiments the extent to which these results reflect the behavior of the preconditioner in more realistic scenarios, that is, for Dirichlet boundary conditions or non-constant wind. Our observations are that the convergence behavior of Krylov subspace methods is independent of $\nu$ for the Dirichlet problem with constant wind and mildly dependent on $\nu$ for variable wind; convergence also depends mildly on the mesh size $h$, with iteration counts increasing in proportion to $h^{-1 / 2}$. These conclusions also hold for variants of the BFBt preconditioner designed to keep computational costs low. In particular, use of $\mathcal{Q}$ with (8) in an iteration entails two Poisson solves on the pressure space and (for any $X)$ the solution of a set of convection-diffusion equations on the velocity space. We show that these computations can be approximated using inner iterations with little degradation of performance of the outer iteration. In $\S 5$ we make some summarizing remarks, and in an appendix we present a partial analysis of the behavior of the BFBt preconditioner for the Stokes problem.

2. Finite difference discretization. We briefly describe the MAC finite difference scheme. Assume that $\Omega$ is the rectangular region $(0,1) \times(0,1)$, divided into a uniform $n \times n$ grid of cells of width $h=1 / n$. Let $\mathbf{u}=(u, v)^{T}$ denote the velocity field and $\mathbf{w}=(a, b)^{T}$ the wind. The discrete velocities and pressures are defined on a staggered grid in which the discrete values of $u$ lie in the centers of the cell boundaries orthogonal to the $x$-axis, the discrete values of $v$ lie in the center of the cell boundaries orthogonal to the $y$-axis, and the discrete pressures lie in the cell centers. An example is shown in Fig. 1.

The matrix of (3) contains three block rows, the first two of which come from the momentum equations for the individual components of the discrete velocity field and 


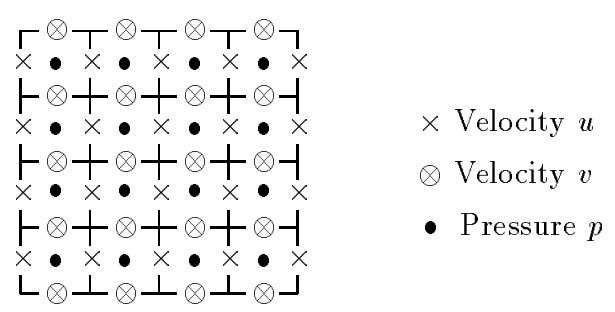

FIG. 1. Staggered grid for the MAC finite difference discretization.

the last to the incompressibility constraint. $F$ and $B$ have the form

$$
F=\left(\begin{array}{cc}
F_{1} & 0 \\
0 & F_{2}
\end{array}\right), \quad B=\left(\begin{array}{ll}
B_{1} & B_{2}
\end{array}\right),
$$

where $F_{i}=\nu A_{i}+N_{i}$. The submatrices are defined as follows. Let $\phi_{j k}$ denote the value of a mesh function $\phi$ at the point $(j h, k h) \in \bar{\Omega}$. The form of the indices $(j, k)$ depends on the mesh function to which they correspond; in particular, they need not be integers. The first block row of $(3)$ is defined by

$$
\begin{aligned}
{[-\Delta u]_{j k} \approx\left[A_{1} u\right]_{j k} } & \equiv \frac{1}{h^{2}}\left(4 u_{j k}-u_{j-1, k}-u_{j+1, k}-u_{j, k+1}-u_{j, k-1}\right) \\
{\left[a u_{x}\right]_{j k} \approx\left[N_{1}^{(x)} u\right]_{j k} } & \equiv \frac{1}{2 h}\left(a_{j+1 / 2, k} u_{j+1, k}-a_{j-1 / 2, k} u_{j-1, k}\right) \\
{\left[b u_{y}\right]_{j k} \approx\left[N_{1}^{(y)} u\right]_{j k} } & \equiv \frac{1}{2 h}\left(b_{j, k+1 / 2} u_{j, k+1}-b_{j, k-1 / 2} u_{j, k-1}\right) \\
{\left[p_{x}\right]_{j k} \approx\left[B_{1}^{T} p\right]_{j k} } & \equiv \frac{1}{h}\left(p_{j+1 / 2, k}-p_{j-1 / 2, k}\right) .
\end{aligned}
$$

The second block row (associated with $v$ ) is defined analogously. The discrete convection operator $N_{1}=N_{1}^{(x)}+N_{1}^{(y)}$ represents a second-order approximation to

$$
\frac{1}{2}[\mathbf{w} \cdot \operatorname{grad} u+\operatorname{div}(u \mathbf{w})]
$$

this is a skew-self-adjoint version of the first convection term of (2). ${ }^{1}$ The second term is treated analogously, and the resulting matrix $N$ is skew-symmetric. The discrete incompressibility constraint is

$(10)-\left[u_{x}+v_{y}\right]_{j k} \approx[B \mathbf{u}]_{j k}=-\left[\frac{1}{h}\left(u_{j+1 / 2, k}-u_{j-1 / 2, k}\right)+\frac{1}{h}\left(v_{j, k+1 / 2}-v_{j, k-1 / 2}\right)\right]$.

We will discuss the treatment of boundary conditions in Sections 3 and 4.

3. Fourier analysis. Suppose (2) is posed with constant wind $\mathbf{w}$ and periodic boundary conditions

$$
\mathbf{u}(x, 0)=\mathbf{u}(x, 1), \quad \mathbf{u}(0, y)=\mathbf{u}(1, y)
$$

For the discretization, indexing in (9) and (10) is done in mod $n$ arithmetic. In particular, mesh points on the left and right (or top and bottom) boundaries are

\footnotetext{
1 This form of the convection operator leads to the skew-self-adjoint weak form used, for example, in $[14$, p. 53$],[26$, p. 205].
} 
identified, and if $(j, k)$ corresponds to a pressure mesh point next to the boundary (e.g., $j=1 / 2$ ) then one of its "neighboring" points is next to the opposite boundary $(j=n-1 / 2)$. The discrete versions of $u, v$ and $p$ each contain $n^{2}$ components, and therefore each of

$$
F_{1}, F_{2}, B_{1}, B_{2}, B_{1}^{T}, B_{2}^{T}
$$

is a square matrix of order $n^{2}$. We also define a discrete convection-diffusion operator $F_{p}$ on the pressure space using the first three terms of $(9)$. That is,

$$
F_{p} \equiv \nu A+N
$$

where $A$ and $N=N^{(x)}+N^{(y)}$ are specified exactly as in $(9)$ and $(j, k)$ now corresponds to indices for grid functions in the pressure space. An analogous idea is used in the definition of "distributive relaxation" schemes for multigrid methods applied to the Stokes and Navier-Stokes equations, see [3, 30]. $F_{p}$ is also defined using periodic boundary conditions for the discrete pressures. It is then straightforward to prove the following lemma by direct calculation.

LeMma 3.1. If $\mathbf{w}$ is a constant vector then $F B^{T}=B^{T} F_{p}$.

We will assume for the rest of this section that the wind $\mathrm{w}$ is constant on $\Omega$. The preconditioned operator can then be analyzed using Fourier techniques of the type described in [4]. It turns out that $F_{1}=F_{2}=F_{p}$ in this case and in some of the discussion we will refer to them collectively as $F_{*}$. Consider the discrete exponential mesh functions $\left\{\psi^{(s, t)} \mid 0 \leq s, t<n\right\}$ where

$$
\left[\psi^{(s, t)}\right]_{j k}=e^{2 \pi i s x_{j}} e^{2 \pi i t y_{k}}, \quad x_{j}=j / n, y_{k}=k / n, \quad 0 \leq j, k<n .
$$

These make up an orthogonal basis for $\mathbf{C}^{n^{2}}$. The matrices associated with the periodic problem satisfy

$$
\begin{aligned}
& F_{*} \psi^{(s, t)}=\left(\nu \frac{4\left(\sin ^{2}(\pi s h)+\sin ^{2}(\pi t h)\right)}{h^{2}}+i \frac{w_{1} \sin (2 \pi s h)+w_{2} \sin (2 \pi t h)}{h}\right) \psi^{(s, t)}, \\
& B_{1} \psi^{(s, t)}=\frac{1}{h}\left(1-e^{2 \pi i s h}\right) \psi^{(s, t)}, \quad B_{1}^{T} \psi^{(s, t)}=\frac{1}{h}\left(1-e^{-2 \pi i s h}\right) \psi^{(s, t)}, \\
& B_{2} \psi^{(s, t)}=\frac{1}{h}\left(1-e^{2 \pi i t h}\right) \psi^{(s, t)}, \quad B_{2}^{T} \psi^{(s, t)}=\frac{1}{h}\left(1-e^{-2 \pi i t h}\right) \psi^{(s, t)} .
\end{aligned}
$$

That is, the mesh functions of (12) constitute an orthogonal basis of eigenvectors for each of these matrices. The particular choice $s=t=0$ leads to $\psi^{(0,0)} \equiv 1$, with an associated eigenvalue equal to 0 for all the matrices of (11). Let $\mathcal{S}_{0}$ denote the space generated by $\psi^{(0,0)}$ and let $\mathcal{S}_{0}^{\perp}$ denote the orthogonal complement of $\mathcal{S}_{0}$. It can be verified that all eigenvalues of $F_{*}$ are nonzero for other combinations of $s$ and $t$, so that this matrix represents a nonsingular operator from $\mathcal{S}_{0}^{\perp}$ to itself. We can also extend $F_{*}^{-1}$ to an operator on all of $\mathbf{C}^{n^{2}}$ by defining it to be 0 on $\mathcal{S}_{0}^{\perp}$. Therefore, it makes sense to consider the operators

$$
F^{-1} B^{T}, \quad B^{T} F_{p}^{-1}, \quad B F^{-1} B^{T}=B_{1} F_{1}^{-1} B_{1}^{T}+B_{2} F_{2}^{-1} B_{2}^{T} .
$$

Lemma 3.2. The Schur complement $B F^{-1} B^{T}$ of (14) is a nonsingular operator from $\mathcal{S}_{0}^{\perp}$ to itself. 
Proof. It follows immediately from (13) that

$$
B F^{-1} B^{T} \psi^{(s, t)}=\lambda_{s t} \psi^{(s, t)}
$$

where

$$
\lambda_{s t}=\frac{4\left(\sin ^{2}(\pi s h)+\sin ^{2}(\pi t h)\right)}{\nu\left[4\left(\sin ^{2}(\pi s h)+\sin ^{2}(\pi t h)\right)\right]+i h\left(w_{1} \sin (2 \pi s h)+w_{2} \sin (2 \pi t h)\right)} .
$$

This eigenvalue is nonzero if $s \neq 0$ or $t \neq 0$. $\square$

Theorem 3.3. Let $X$ be given by (8). If $B F^{-1} B^{T}, X$ and (the identity) $I$ are viewed as operators from $\mathcal{S}_{0}^{\perp}$ to itself, then $X$ is simply an alternative representation of $B F^{-1} B^{T}$, and $B F^{-1} B^{T} X^{-1}=I$.

Proof. It follows from Lemma 3.1 that $F^{-1} B^{T}=B^{T} F_{p}^{-1}$. Consequently,

$$
\operatorname{range}\left(B^{T}\right)=\operatorname{range}\left(B^{T} F_{p}^{-1}\right)=\operatorname{range}\left(F^{-1} B^{T}\right) \text {, }
$$

so that ( 7 ) holds. The assertion follows from the discussion of $\xi 1$.

4. Experiments on Dirichlet problems. The results of $\S 3$ show that (8) defines a perfect preconditioner for constant wind and periodic boundary conditions. In this section, we examine the performance of the BFBt preconditioner and some variants on more realistic problems with Dirichlet boundary conditions $\mathbf{u}=\mathbf{g}$ on $\partial \Omega$.

For most of the experiments, the Oseen equations are posed on $\Omega=(0,1) \times(0,1)$ and discretized with the MAC scheme. This is defined as in $\S 2$ except when the discrete operators refer to grid indices outside $\bar{\Omega}$ (for example, in components of the first discrete momentum equation centered at the values of $u$ next to the bottom of $\partial \Omega$ ). Linear extrapolation is used in these cases; see the definition of $T_{E}$ in $\S$ A.2 for details. We consider two sets of coefficients, constant $\mathbf{w}=(1,2)$ and $\mathbf{w}=$ a circular vortex. In the latter case $\mathbf{w}$ is the image of

$$
\left(2 y\left(1-x^{2}\right),-2 x\left(1-y^{2}\right)\right)
$$

under the linear mapping from $(-1,1) \times(-1,1)$ to $\Omega$. Rather than impose explicit boundary conditions, we eliminate the discrete velocities on the boundary from the system and use a normally distributed random vector with mean 0 and variance 1 for the right hand side $\mathbf{f}$ of (3). We also demonstrate that the BFBt methodology is not restricted to finite differences with a set of experiments for a finite element discretization of the driven cavity problem. Both discretizations satisfy an inf-sup condition [14].

Our results are in the form of iteration counts for various combinations of $\nu$ and $h$. We restrict our attention to values of $\nu$ such that for the smallest mesh size considered, $\frac{\mathrm{w} h}{2 \nu}$ is of order 1 , so that the discretizations are reasonably accurate. Unless otherwise indicated, the iterative solver is GMRES with right-oriented preconditioning; the initial guess for all tests is $\mathbf{u}_{0}=0, p_{0}=0$, and the stopping criterion is

$$
\frac{\left\|\boldsymbol{f}-\mathcal{A} \boldsymbol{x}_{k}\right\|_{2}}{\|\boldsymbol{f}\|_{2}} \leq 10^{-6}
$$

where $f$ and $\boldsymbol{x}_{k}$ denote the right hand side and iterate for the block system (3).

We now present the results for the BFBt preconditioner. For comparison we show analogous iteration counts for the preconditioner (4) where $X=\frac{1}{\nu} M$ is the diagonal 
TABLE 1

Iterations of GMRES for constant wind $w=(1,2)$ with finite difference discretization.

\begin{tabular}{c|ccc|ccc} 
& \multicolumn{3}{|c|}{$X=X_{\text {BFBt }}$} & \multicolumn{3}{c}{$X=\frac{1}{\nu} M$} \\
\hline \multicolumn{1}{c|}{} & $1 / 16$ & $1 / 32$ & $1 / 64$ & $1 / 16$ & $1 / 32$ & $1 / 64$ \\
\hline$\nu=1$ & 9 & 10 & 12 & 12 & 10 & 10 \\
$\nu=1 / 10$ & 8 & 11 & 15 & 34 & 34 & 33 \\
$\nu=1 / 30$ & 9 & 10 & 13 & 88 & 87 & 83 \\
$\nu=1 / 50$ & 9 & 10 & 11 & 144 & 145 & 139
\end{tabular}

TABLE 2

Iterations of GMRES for $w=$ circular vortex with finite difference discretization.

\begin{tabular}{c|ccc|ccc} 
& \multicolumn{3}{|c|}{$X=X_{\text {BFBt }}$} & \multicolumn{3}{c}{$X=\frac{1}{\nu} M$} \\
\hline \multicolumn{1}{c|}{$1 / 16$} & $1 / 32$ & $1 / 64$ & $1 / 16$ & $1 / 32$ & $1 / 64$ \\
\hline$\nu=1$ & 8 & 10 & 12 & 10 & 10 & 10 \\
$\nu=1 / 10$ & 11 & 14 & 18 & 19 & 19 & 18 \\
$\nu=1 / 30$ & 14 & 17 & 21 & 47 & 46 & 43 \\
$\nu=1 / 50$ & 16 & 18 & 23 & 79 & 77 & 73
\end{tabular}

of the scaled mass matrix as described in [5]; $X=\frac{1}{\nu}\left(h^{2} I\right)$ for the MAC scheme. For the moment we are ignoring any issues of cost. ${ }^{2}$

We present four sets of results. For the MAC discretization, Table 1 shows the number of iterations for convergence of GMRES for constant wind, and Table 2 shows the results for the circular vortex. Table 3 shows analogous statistics for one example of a different discretization consisting of bilinear finite elements for both velocities and pressures, with the pressure grid of width $2 h$ and streamline upwinding for the velocities. ${ }^{3}$ To show the effects of a suboptimal Krylov subspace iteration, Table 4 shows the iteration counts when the BFBt preconditioner is combined with quasiminimum residual (QMR) iteration without look-ahead [10], which (in contrast to GMRES) has a fixed cost per step.

As defined, the BFBt preconditioner requires several costly subsidiary computations. For each step of GMRES, the preconditioning entails the action of $\mathcal{Q}^{-1}$ where $\mathcal{Q}$ is given by (4). It can be seen from the factorization

$$
\mathcal{Q}^{-1}=\left(\begin{array}{cc}
F^{-1} & 0 \\
0 & I
\end{array}\right)\left(\begin{array}{cc}
I & B^{T} \\
0 & -I
\end{array}\right)\left(\begin{array}{cc}
I & 0 \\
0 & X^{-1}
\end{array}\right)
$$

that computing the action of $\mathcal{Q}^{-1}$ entails

- computing the action of $X^{-1}$;

- performing a matrix-vector product by $B^{T}$; and

\footnotetext{
${ }^{2}$ All computations were performed in MATLAB on either a Sun SPARC-20 workstation or a DEC-Alpha $21004 / 275$ workstation. For both preconditioners, the action of $F^{-1}$ was computed using Gaussian elimination. For the BFBt preconditioner the action of $\left(B B^{T}\right)^{-1}$ was computed by direct methods using the pseudo-inverse of $B B^{T}$, except when $h=1 / 64$ for the MAC discretization; in that case, this computation was done using a multigrid iteration in which the relative residual is forced to be less than $10^{-8}$.

${ }^{3}$ The test problem is slightly different here. It is posed on $\Omega=(-1,1) \times(-1,1)$ with w as in $(15)$, $\mathbf{f}=0$ in (1), and boundary conditions $u=v=0$ when $x= \pm 1$ or $y=-1$, and $u=1$, $v=0$ when $y=1$. See [5] for more details. Some of the entries in Table 3 are taken from [5].
} 
TABLE 3

Iterations of GMRES for $w=$ circular vortex with bilinear finite element discretization.

\begin{tabular}{c|ccc|ccc} 
& \multicolumn{3}{|c|}{$X=X_{\text {BFBt }}$} & \multicolumn{3}{c}{$X=\frac{1}{\nu} M$} \\
\hline \multicolumn{1}{c|}{$h$} & $2 / 16$ & $2 / 32$ & $2 / 64$ & $2 / 16$ & $2 / 32$ & $2 / 64$ \\
\hline$\nu=1$ & 7 & 9 & 11 & 21 & 22 & 21 \\
$\nu=1 / 10$ & 10 & 12 & 15 & 32 & 36 & 35 \\
$\nu=1 / 30$ & 13 & 15 & 17 & 44 & 56 & 64 \\
$\nu=1 / 50$ & 15 & 17 & 19 & 48 & 72 & 97
\end{tabular}

TABLE 4

Iterations of QMR with finite difference discretization and BFBt preconditioning.

\begin{tabular}{c|ccc|ccc} 
& \multicolumn{3}{|c|}{$w=(1,2)$} & \multicolumn{3}{c}{ circular vortex } \\
\hline$h$ & $1 / 16$ & $1 / 32$ & $1 / 64$ & $1 / 16$ & $1 / 32$ & $1 / 64$ \\
\hline$\nu=1$ & 9 & 13 & 15 & 9 & 12 & 15 \\
$\nu=1 / 10$ & 9 & 13 & 17 & 12 & 17 & 21 \\
$\nu=1 / 30$ & 10 & 11 & 14 & 14 & 20 & 27 \\
$\nu=1 / 50$ & 10 & 12 & 13 & 16 & 21 & 28
\end{tabular}

- computing the action of $F^{-1}$.

$B B^{T}$ is a discrete Poisson operator on the pressure space (see $\S$ A.2 for a derivation for the MAC scheme), so that for the BFBt preconditioner, computing the action of $X^{-1}$ entails solving two discrete Poisson equations. These together with the convectiondiffusion solves are potentially expensive operations, and the BFBt preconditioner is significantly more costly than when $X=\frac{1}{\nu} M$, a diagonal matrix. We now examine what happens when less costly computations based on inner iteration are used in place of these three operations. We consider only finite differences here although the same methodologies are applicable to other discretizations. These versions of the preconditioner also require less storage, and in particular this enabled us to further explore some trends in the data with a finer mesh size $h=1 / 128$ and smaller viscosity parameter $\nu=1 / 100$ (used only with this fine mesh).

We first consider the effect of replacing the Poisson solves with approximations derived from one step of V-cycle multigrid [19, Ch. 1]. ${ }^{4}$ That is, the modified BFBt preconditioner uses

$$
X=S_{M G}\left(B F B^{T}\right) S_{M G},
$$

where $S_{M G}$ is the multigrid approximation to $\left(B B^{T}\right)^{-1}$. The computational costs of this algorithm are of the same order of magnitude as when $X=\frac{1}{\nu} M$. (We are still solving the convection-diffusion equations exactly except when $h=1 / 128$; in this case, we use an iterative method based on relaxation and force the relative residual norm to be less than $10^{-8}$.) The iteration counts for the two sets of benchmark problems are shown in Tables 5 and 6 .

Next, we consider the effect of also replacing the convection-diffusion solves with approximate solutions derived from iterative methods. We will comment on the choice of method below. The performance of any such method will depend on the relative

\footnotetext{
${ }^{4}$ The multigrid computation used damped Jacobi smoothing with optimal smoothing parameter $\omega=4 / 5$, one pre-smoothing and one post-smoothing step, and bilinear interpolation for prolongation.
} 
TABLE 5

Iterations of GMRES with modified BFBt preconditioning using multigrid for the Poisson equation. Wind $w=(1,2)$, finite difference discretization.

\begin{tabular}{l|cccc} 
& \multicolumn{4}{|c}{$X=X_{\text {BFBt } / M G}$} \\
\hline \multicolumn{1}{c|}{$h$} & $1 / 16$ & $1 / 32$ & $1 / 64$ & $1 / 128$ \\
\hline$\nu=1$ & 11 & 12 & 15 & 19 \\
$\nu=1 / 10$ & 12 & 13 & 17 & 22 \\
$\nu=1 / 30$ & 12 & 12 & 15 & 20 \\
$\nu=1 / 50$ & 13 & 13 & 14 & 18 \\
$\nu=1 / 100$ & & & & 14
\end{tabular}

TABLE 6

Iterations of GMRES with modified BFBt preconditioning using multigrid for the Poisson equation. Wind $w=$ circular vortex, finite difference discretization.

\begin{tabular}{c|ccc} 
& \multicolumn{3}{|c}{$X=X_{B F B t / M G}$} \\
\hline \multicolumn{1}{c|}{} & $1 / 16$ & $1 / 32$ & $1 / 64$ \\
\hline$\nu=1$ & 11 & 12 & 15 \\
$\nu=1 / 10$ & 14 & 16 & 20 \\
$\nu=1 / 30$ & 19 & 21 & 24 \\
$\nu=1 / 50$ & 21 & 24 & 27
\end{tabular}

amount of convection and diffusion in the problem, i.e., the value of $\nu$ [7]. Therefore, rather than use a fixed number of iterations, we perform the inner iteration until the stopping criterion

$$
\frac{\left\|w-F v_{k}\right\|_{2}}{\|w\|_{2}} \leq \tau=10^{-2}
$$

is satisfied, where $w$ represents the right hand side for each convection-diffusion equation and $v_{0}=0$ is the initial guess. The number of these iterations may vary from step to step of the outer GMRES iteration, so that we are no longer using a fixed preconditioner $\mathcal{Q}$; instead we have a series of operators $\mathcal{Q}_{k}$ that vary with the GMRES step. This may cause difficulties for GMRES, but these can be avoided with a "flexible" variant of GMRES (FGMRES) designed for this situation [23], which we use in these tests. The two algorithms are mathematically equivalent when $\mathcal{Q}_{k}$ is fixed. The results for constant wind are shown in Table 7 , and for the circular vortex in Table 8. We also present the performance using the scaled mass matrix. A dash "-" indicates failure to converge; this was due to divergence of the iteration for the convection-diffusion equation and is an artifact of inaccuracy of the discretization.

We highlight some trends displayed by these data as follows:

1. In all tests with the constant coefficient problem (Tables 1, 4, and 7, left, and Table 5), iteration counts with the BFBt preconditioner are independent of the viscosity $\nu$; indeed, in some cases they actually decrease with $\nu$. For the circular vortex, however, the iteration counts appear not to be independent of $\nu$, instead exhibiting some growth as $\nu$ decreases (Tables 2 and 8, left, Table 4, right, and Table 6).

2. In all cases, the counts with BFBt preconditioning increase slowly with $h^{-1}$.

3. The iteration counts for $X=\frac{1}{\nu} M$ are independent of $h^{-1}$ but they grow roughly linearly with $1 / \nu$; these trends are consistent with the analysis of [5]. 
TABLE 7

Iterations of FGMRES with modified BFBt preconditioning using multigrid for the Poisson equation and iteration for the convection-diffusion equation. Wind $w=(1,2)$, finite difference discretization.

\begin{tabular}{c|cccc|cccc} 
& \multicolumn{4}{|c|}{$X=X_{\text {BFBt/MG/Iter }}$} & \multicolumn{4}{c}{$X=\frac{1}{\nu} M$} \\
\hline \multicolumn{1}{c|}{$h$} & $1 / 16$ & $1 / 32$ & $1 / 64$ & $1 / 128$ & $1 / 16$ & $1 / 32$ & $1 / 64$ & $1 / 128$ \\
\hline$\nu=1$ & 11 & 13 & 16 & 20 & 12 & 11 & 12 & 12 \\
$\nu=1 / 10$ & 12 & 14 & 17 & 22 & 35 & 34 & 33 & 32 \\
$\nu=1 / 30$ & 12 & 13 & 15 & 20 & 111 & 88 & 85 & 85 \\
$\nu=1 / 50$ & - & 13 & 14 & 18 & - & 185 & 141 & 142 \\
$\nu=1 / 100$ & & & & 15 & & & &
\end{tabular}

TABLE 8

Iterations of FGMRES with modified BFBt preconditioning using multigrid for the Poisson equation and iteration for the convection-diffusion equation. Wind $w=$ circular vortex, finite difference discretization.

\begin{tabular}{l|cccc|cccc} 
& \multicolumn{4}{|c|}{$X=X_{\text {BFBt } / M G / \text { Iter }}$} & \multicolumn{4}{c}{$X=\frac{1}{\nu} M$} \\
\hline \multicolumn{1}{c|}{$h$} & $1 / 16$ & $1 / 32$ & $1 / 64$ & $1 / 128$ & $1 / 16$ & $1 / 32$ & $1 / 64$ & $1 / 128$ \\
\hline$\nu=1$ & 11 & 13 & 16 & 19 & 11 & 12 & 12 & 12 \\
$\nu=1 / 10$ & 14 & 16 & 20 & 25 & 19 & 19 & 19 & 18 \\
$\nu=1 / 30$ & 19 & 21 & 24 & 31 & 51 & 45 & 44 & 43 \\
$\nu=1 / 50$ & - & 28 & 27 & 34 & - & 95 & 73 & 73 \\
$\nu=1 / 100$ & & & & 37 & & & & 155
\end{tabular}

The counts are smaller for the circular vortex than for the constant wind; we have no explanation for this. However, the same qualitative patterns are present for both problems. In practical situations it is often desired to compute solutions of a fixed accuracy for a variety of values of $\nu$ by letting $h \rightarrow 0$ and $\nu \rightarrow 0$ simultaneously; with respect to this criterion, the BFBt preconditioner requires significantly fewer iterations as the viscosity decreases.

4. The results for bilinear finite elements (Table 3 ) are qualitatively the same as for finite differences.

5. The iteration counts of QMR (Table 4) are only slightly higher than those of GMRES and they display the same patterns. This was also true for $X=\frac{1}{\nu} M$ (results not shown).

6. Comparison of Tables 5 and 6 with Tables 1 and 2 shows that replacing the exact Poisson solve with one multigrid step leads to little increase in iteration counts (less than $25 \%$ for $h=1 / 64$ ). The same trends with respect to $\nu$ and $h$ are evident, and these carry over to $h=1 / 128$. Note that the multigrid method used here is not necessarily an optimal choice, just a simple one.

7. Approximate solution of the convection-diffusion problem produces little degradation in performance of either preconditioner, even though the tolerance $\tau$ in $(16)$ is very mild.

We return to the first two items on this list, the dependence of iteration counts with BFBt preconditioning on the parameters $\nu$ (for the circular vortex) and $h$. To try to get a feeling for this dependence, we plot the behavior of the solver along with graphs of functions that might model this behavior. In particular, Figure 2 plots iteration counts as a function of $\nu^{-1}$ using the entries of Table 8 for $h=1 / 128$, 
FIG. 2. Comparison of iteration counts with various functions of $\nu$, for $h=1 / 128$.

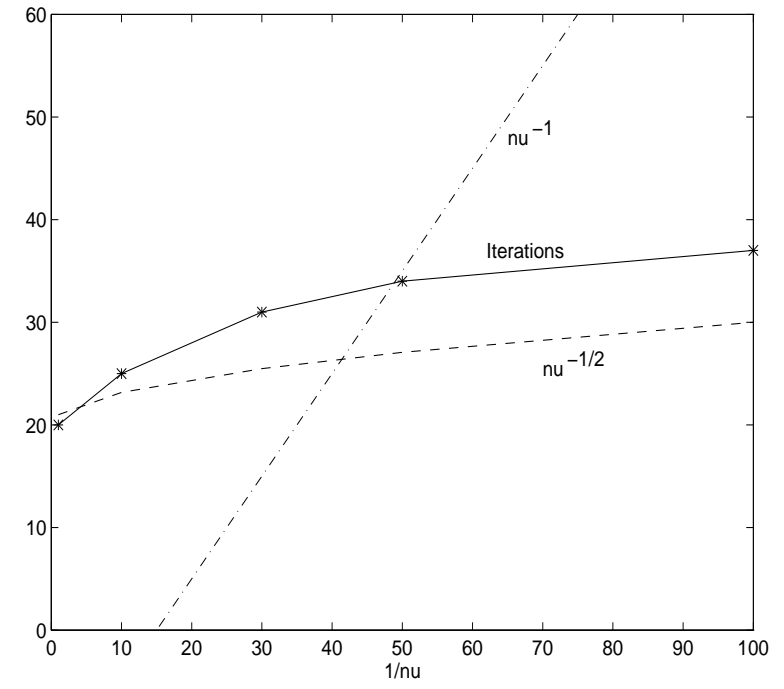

together with graphs of the functions $\nu^{-1}$ and $\nu^{-1 / 2}$; the latter curves were shifted to make the figure easy to view. These results suggest that dependence on $\nu$ is like $O\left(\nu^{-1 / 2}\right)$, although it is difficult to make a precise statement with this limited amount of data. Similarly, Figure 3 plots iteration counts as a function of $h^{-1}$ using the entries from Table 8 for $\nu=1 / 30$, and these are compared with $h^{-1}$ and $h^{-1 / 2}$. These results typify the behavior for all the values $\nu$ in the table and suggest that the dependence on $h$ is of order $h^{-1 / 2}$; see also $\S \mathrm{A} .2$.

We comment briefly on the convection-diffusion solvers. For simple flows, it is fairly easy to construct relaxation strategies that follow the flow and converge rapidly [7], but for more complex flows, especially with recirculations, this is a more difficult task. In these tests, we used a horizontal 1-line SOR iteration with relaxation parameter given by the optimal choice for the constant coefficient problem, which can be computed analytically [8]. We used this for the circular wind only for convenience of coding, and convergence was slow for these problems. Many other options for solving this problem (such as multigrid) are available. We also ran some tests with another inner iteration (using a "multidirectional relaxation") and found the number of outer iterations not to depend significantly on the choice of the method used here. This points to the importance of the convection-diffusion problem for the BFBt preconditioner; it is critical that that the approximate solution to this subproblem be computed efficiently for the complete computation to be inexpensive.

5. Concluding remarks. We summarize this study as follows. The main new result is that the performance of the BFBt preconditioner for solving the steadystate Oseen equations with Dirichlet boundary conditions depends very mildly on the viscosity $\nu$. This stands in contrast to other preconditioning methods, where performance deteriorates more dramatically as $\nu \rightarrow 0$. Its performance depends on the mesh size $h$ with iterations apparently growing in proportion to $h^{-1 / 2}$. If a series of Dirichlet problems with decreasing viscosity are to be solved in such a way that $h$ is proportional to $\nu$, then we expect the iteration counts to grow like $O\left(\nu^{-1 / 2}\right)$.

This conclusion also appears to hold for "inexact" versions of the BFBt preconditioner in which the expensive subsidiary computations, solution of the Poisson equation 
FIG. 3. Comparison of iteration counts with various functions of $h$, for $\nu=1 / 30$.

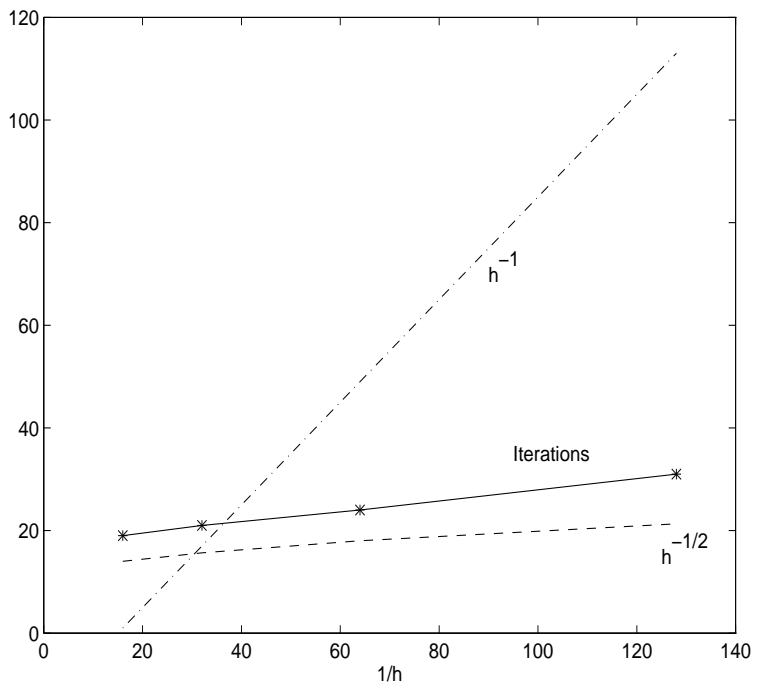

and convection-diffusion equation, are replaced by iterative solves with mild stopping criteria. The effectiveness of these variants of the BFBt preconditioner depends on having efficient methods for the convection-diffusion equation.

This issue is also a critical one for multigrid methods for the Navier-Stokes equations $[3,27,28,30]$. It is difficult to make a simple comparison between the methods proposed here and multigrid; most reported studies show a dependence on viscosity (or Reynolds number) but not on mesh size [27, 28, 30]. An advantage of the BFBt preconditioner is that it can be applied easily to systems arising from mixed finite elements with different grids for velocities and pressure (as shown in Table 3).

If the goal is to solve a fixed Dirichlet problem on a sequence of finer meshes, then there are other methods whose performance is less dependent on the mesh size [5, 11]. This is true in particular for the Stokes equations, where several methods exist whose convergence rate is independent of $h[1,2,22,25]$.

Finally, we note that the boundary conditions are very important here. Fourier analysis is often used as a guideline in behavior of numerical methods [21], and there are numerous examples of cases where the results for periodic boundary conditions are predictive of performance for Dirichlet conditions [4]. However, here there is a qualitative difference in the two types of problems. See [18] for other examples of the effects of boundary conditions on iterative methods.

\section{A. Appendix: Partial analysis for the preconditioned Stokes problem.} We show that the minimum eigenvalue for the BFBt-preconditioned Stokes operator is no smaller than one and give a partial analysis that suggests why its maximum eigenvalue is proportional to $h^{-1}$.

A.1. Lower bound. Suppose the coefficient matrix of (3) is a discrete Stokes operator, i.e., it is derived from $\nu=1, \mathrm{w}=0$ in (2), with Dirichlet boundary conditions. Therefore, $F=A$. Let the singular value decomposition of $B$ be denoted

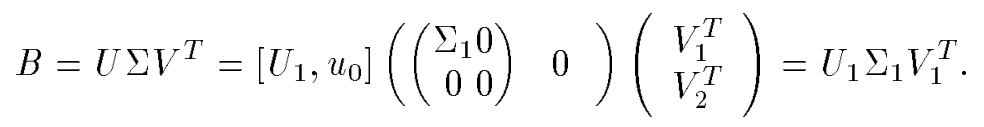


The columns of $U_{1}$ span $\operatorname{range}(B)$, the columns of $V_{1}$ span $\operatorname{range}\left(B^{T}\right)$, and $u_{0}$ is parallel to the discrete hydrostatic pressure $p \equiv 1$, which determines the null space $\mathcal{S}_{0}$ of $B^{T}$. It follows that

$$
B B^{T}=U_{1} \Sigma_{1}^{2} U_{1}^{T}
$$

As in $\S 3$, we will treat $B^{T}$ as an operator defined on $\mathcal{S}_{0}^{\perp}$; on this space $B B^{T}$ and $B A B^{T}$ represent nonsingular operators whose inverses are given by the matrix pseudo-inverses

$$
\begin{array}{ll}
\left(B B^{T}\right)^{-1} & =U_{1} \Sigma_{1}^{-2} U_{1}^{T}, \\
\left(B A B^{T}\right)^{-1} & =U_{1} \Sigma_{1}^{-1}\left(V_{1}^{T} A V_{1}\right)^{-1} \Sigma_{1}^{-1} U_{1}^{T} .
\end{array}
$$

We are interested in the minimum eigenvalue of the generalized eigenvalue problem

$$
\left(B A^{-1} B^{T}\right) p=\lambda\left(B B^{T}\right)\left(B A B^{T}\right)^{-1}\left(B B^{T}\right) p .
$$

The Schur complement matrix on the left is

$$
S \equiv B A^{-1} B^{T}=U_{1} \Sigma_{1} V_{1}^{T} A^{-1} V_{1} \Sigma_{1} U_{1}^{T},
$$

and the preconditioning matrix on the right is

$$
X=U_{1} \Sigma_{1}\left(V_{1}^{T} A V_{1}\right)^{-1} \Sigma_{1} U_{1}^{T} .
$$

Consequently,

$$
S=X+U_{1} \Sigma_{1}\left[V_{1}^{T} A^{-1} V_{1}-\left(V_{1}^{T} A V_{1}\right)^{-1}\right] \Sigma_{1} U_{1}^{T} .
$$

Using this splitting of $S$, we can prove the following result.

THEOREM A.1. The minimum eigenvalue of the preconditioned generalized Stokes operator with Dirichlet boundary conditions is $\lambda=1$.

Proof. We will show that $V_{1}^{T} A^{-1} V_{1}-\left(V_{1}^{T} A V_{1}\right)^{-1}$ is positive semi-definite. Let

$$
D=V^{T} A V=\left(\begin{array}{ll}
D_{11} & D_{12} \\
D_{21} & D_{22}
\end{array}\right)
$$

where $D_{i j}=V_{i}^{T} A V_{j} . D$ is symmetric positive-definite and standard analysis gives

$$
D=\left(\begin{array}{cc}
I & 0 \\
D_{21} D_{11}^{-1} & I
\end{array}\right)\left(\begin{array}{cc}
D_{11} & 0 \\
0 & D_{22}-D_{21} D_{11}^{-1} D_{12}
\end{array}\right)\left(\begin{array}{cc}
I & D_{11}^{-1} D_{12} \\
0 & I
\end{array}\right) \text {. }
$$

Letting $C_{D} \equiv D_{22}-D_{21} D_{11}^{-1} D_{12}$, this in turn implies that

$$
D^{-1}=\left(\begin{array}{cc}
D_{11}^{-1}+D_{11}^{-1} D_{12} C_{D}^{-1} D_{21} D_{11}^{-1} & -D_{11}^{-1} D_{12} C_{D}^{-1} \\
-C_{D}^{-1} D_{21} D_{11}^{-1} & C_{D}^{-1}
\end{array}\right) .
$$

But $D^{-1}=V^{T} A^{-1} V$, so that

$$
V_{1}^{T} A^{-1} V_{1}=\left[D^{-1}\right]_{11}=D_{11}^{-1}+D_{11}^{-1} D_{12} C_{D}^{-1} D_{21} D_{11}^{-1} .
$$


FIG. 4. Maximum eigenvalue for the Stokes problem with BFBt preconditioner.

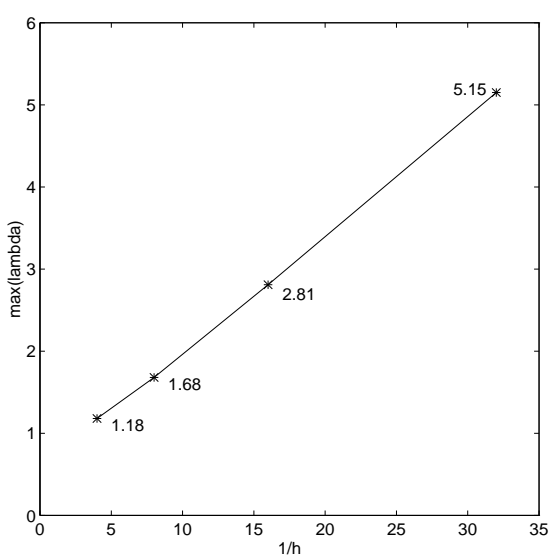

Therefore,

$$
\begin{aligned}
\inf _{x \neq 0} \frac{\left(x, V_{1}^{T} A^{-1} V_{1} x\right)}{\left(x,\left(V_{1} A V_{1}\right)^{-1} x\right)} & =\inf _{x \neq 0} \frac{\left(x, D_{11}^{-1}+D_{11}^{-1} D_{12} C_{D}^{-1} D_{21} D_{11}^{-1} x\right)}{\left(x, D_{11}^{-1} x\right)} \\
& =\inf _{x \neq 0} \frac{\left(x, D_{11}+D_{12} C_{D}^{-1} D_{21} x\right)}{\left(x, D_{11} x\right)} \geq 1 .
\end{aligned}
$$

It follows that $V_{1}^{T} A^{-1} V_{1}-\left(V_{1}^{T} A V_{1}\right)^{-1}$ is positive semi-definite, so that (18) yields

$$
\inf _{q \in \mathcal{S}_{0}^{\perp}} \frac{(q, S q)}{(q, X q)} \geq 1
$$

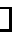

A.2. Largest eigenvalue. The experiments described in $\S 4$ show that convergence rates for the BFBt preconditioner depend on the mesh size. Figure 4 plots the maximum eigenvalue of $(17)$ as a function of $h^{-1}$, where the computations were for $h=1 / 4,1 / 8,1 / 16$ and $1 / 32$. The results indicate that the maximum eigenvalue is of magnitude $O\left(h^{-1}\right)$. By analogy with the standard convergence bounds for symmetric positive definite problems, this suggests that the iteration counts will increase in proportion to $h^{-1 / 2}$, which is consistent with the results of $\S 4$.

To provide some insight into this maximum eigenvalue, we write

$$
\frac{(q, S q)}{(q, X q)}=\frac{(q, S q)}{\left(q,\left(h^{2} I\right) q\right)} \frac{\left(q,\left(h^{2} I\right) q\right)}{(q, X q)}
$$

where $h^{2} I$ is playing the role of the mass matrix for the finite difference discretization. It is well-known [9] that the first quotient on the right is bounded by a constant $\Gamma \leq \sqrt{2}$. We would like a bound for the second quotient of the form

$$
\sup _{q \in \mathcal{S}_{0}^{\perp}} \frac{\left(q,\left(h^{2} I\right) q\right)}{\left(q,\left(B B^{T}\right)\left(B A B^{T}\right)^{-1}\left(B B^{T}\right) q\right)} \leq \frac{c}{h},
$$

or equivalently,

$$
\sup _{q \in \mathcal{S}_{0}^{\perp}} \frac{\left(q, B A B^{T} q\right)}{\left(q,\left(B B^{T}\right)^{2} q\right)} \leq \frac{c}{h^{3}}
$$


The matrices comprising (19) have a tensor product structure derived from onedimensional operators. Let the interval $[0,1]$ be divided into $n$ equally spaced subintervals of width $h=1 / n$, where the $j$ th interval is $\left[x_{j-1}, x_{j}\right]$ with $x_{j}=j h$ and midpoint $\hat{x}_{j}=(j-1 / 2) h$. An example with $n=8$ is shown below.

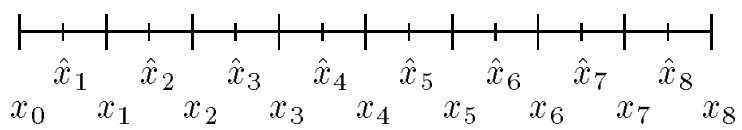

Consider the following three (scaled) finite difference approximations to the onedimensional Laplace operator $-u^{\prime \prime}$ :

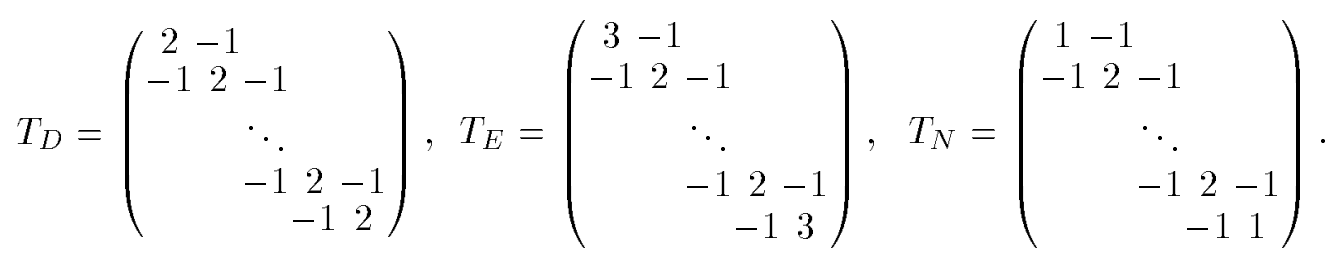

$T_{D}$ and $T_{E}$ are derived from Dirichlet boundary conditions $(u(0), u(1)$ given $)$, and $T_{N}$ is derived from Neumann conditions $\left(u^{\prime}(0), u^{\prime}(1)\right.$ given $) . T_{D}$ is defined at the cell boundaries $\left\{x_{j}\right\}_{j=1}^{n-1}$ and is of order $n-1 ; T_{E}$ and $T_{N}$ are defined at the cell centers $\left\{\hat{x}_{j}\right\}_{j=1}^{n}$ and are of order $n$. In all cases, the discrete Laplace operator at points next to the boundaries depends on the boundary conditions. $T_{E}$ uses linear extrapolation

$$
u\left(\hat{x}_{0}\right)=-u\left(x_{1}\right)+2 u(0), \quad u\left(\hat{x}_{n+1}\right)=-u\left(x_{n}\right)+2 u(1) ;
$$

$T_{N}$ approximates the Neumann boundary conditions as

$$
u^{\prime}(0) \approx\left[u\left(\hat{x}_{1}\right)-u\left(\hat{x}_{0}\right)\right] / h, \quad u^{\prime}(1) \approx\left[u\left(\hat{x}_{n+1}\right)-u\left(\hat{x}_{n}\right)\right] / h .
$$

Note that

$$
T_{E}=T_{N}+2 E_{0}, \quad E_{0}=\left(\begin{array}{cccc}
1 & & & \\
& 0 & & \\
& & \ddots & \\
& & & 0 \\
& & &
\end{array}\right) .
$$

We will also use the difference operator

$$
B_{D}=\left(\begin{array}{rrrr}
-1 & & & \\
1 & -1 & & \\
& \ddots & \ddots & \\
& & 1 & -1 \\
& & & 1
\end{array}\right)
$$

which has dimensions $n \times(n-1)$ and can be viewed as mapping cell boundary grid functions to cell centered functions. It is easily verified that

$$
B_{D} B_{D}^{T}=T_{N}, \quad B_{D} T_{D} B_{D}^{T}=T_{N}^{2} .
$$

Recall that for matrices $X$ and $Y$ where $X$ has dimensions $r \times s$, the tensor product of $X$ and $Y$ is [16, pp. 239ff]

$$
X \otimes Y=\left(\begin{array}{ccc}
x_{11} Y & \cdots & x_{1, s} Y \\
\vdots & \ddots & \vdots \\
x_{r, 1} Y & \cdots & x_{r, s} Y
\end{array}\right) .
$$


Letting $I_{r}$ denote the identity matrix of order $r$, it is straightforward to show that

$$
\begin{aligned}
A & =\left(\begin{array}{cc}
I_{n} \otimes T_{D}+T_{E} \otimes I_{n-1} & 0 \\
0 & I_{n-1} \otimes T_{E}+T_{D} \otimes I_{n}
\end{array}\right), \\
B & =\left[I_{n} \otimes h B_{D}, h B_{D} \otimes I_{n}\right] .
\end{aligned}
$$

The identities (21) then imply

$$
\begin{aligned}
& B B^{T}=h^{2}\left(I_{n} \otimes T_{N}+T_{N} \otimes I_{n}\right), \\
& \left(B B^{T}\right)^{2}=h^{4}\left(I_{n} \otimes T_{N}^{2}+2 T_{N} \otimes T_{N}+T_{N}^{2} \otimes I_{n}\right), \\
& B A B^{T}=h^{2}\left(I_{n} \otimes T_{N}^{2}+T_{E} \otimes T_{N}+T_{N} \otimes T_{E}+T_{N}^{2} \otimes I_{n}\right) .
\end{aligned}
$$

Consequently, relation (19) is equivalent to

$$
\sup _{q \in \mathcal{S}_{0}^{\perp}} \frac{\left(q,\left(I_{n} \otimes T_{N}^{2}+T_{E} \otimes T_{N}+T_{N} \otimes T_{E}+T_{N}^{2} \otimes I_{n}\right) q\right)}{\left(q,\left(I_{n} \otimes T_{N}^{2}+2 T_{N} \otimes T_{N}+T_{N}^{2} \otimes I_{n}\right) q\right)} \leq \frac{c}{h} .
$$

REMARK. This derivation shows that $B B^{T}$ is a scaled discrete Poisson operator on the pressure space with Neuman boundary conditions.

The matrices in (22) differ only by the presence of $T_{E}$ in the cross-terms of the numerator, i.e., the numerator contains submatrices derived from Dirichlet conditions whereas the denominator comes exclusively from Neumann conditions. To get a feeling for why this difference in boundary conditions leads to an inequality of the form (22), consider the generalized eigenvalue problem for the one-dimensional operators,

$$
T_{N} x=\lambda T_{E} x .
$$

It is evident from (20) that there is an eigenvalue $\lambda=1$ of multiplicity $n-2$ for which every eigenvector $x$ satisfies $x_{1}=x_{n}=0$. Moreover, $T_{N} x=0$ for the constant vector $x \equiv 1$, so that 0 is also an eigenvalue. If any $x$ satisfies (23) with $\lambda \neq 0$ and at least one of $x_{1} \neq 0$ or $x_{n} \neq 0$, then the first and last equations (of (23)) imply $\lambda \neq 1$. From the first equation we have

$$
x_{2}-x_{1}=\frac{2 \lambda}{\lambda-1} x_{1}
$$

The assumption $\lambda \neq 1$ together with the (interior) equations $k=2, \ldots, n-1$ imply

$$
x_{k+1}-x_{k}=x_{k}-x_{k-1}=\frac{2 \lambda}{\lambda-1} x_{1}, \quad 2 \leq k \leq n,
$$

where the second equality comes from (24). An inductive argument then yields

$$
x_{k}=\frac{(2 k-1) \lambda-1}{\lambda-1} x_{1}, \quad 2 \leq k \leq n .
$$

Finally, the last equation of (23) implies

$$
x_{n}=\frac{1-\lambda}{2 \lambda}\left(x_{n}-x_{n-1}\right)=-x_{1} .
$$

Equating this expression for $x_{n}$ with the one from (25) yields $\lambda=1 / n=h$. Therefore, we have the following result. 
THEOREM A.2. The eigenvalues of the generalized eigenvalue problem (23) are $\lambda=1$ of multiplicity $n-2, \lambda=0$, and $\lambda=h$. Consequently,

$$
\sup _{x} \frac{\left(x, T_{E} x\right)}{\left(x, T_{N} x\right)}=\frac{1}{h}
$$

where the supremum is taken over all vectors orthogonal to the constant vector.

REMARK. The results of $\S \mathrm{A} .1$ apply in general to any discretization for which the null space is spanned by constant pressures. The discussion of $\S A .2$ applies only for the MAC scheme.

Acknowledgements. The author acknowledges some helpful comments from David Silvester and Robert Pego.

\section{REFERENCES}

[1] J. H. Bramble and J. E. Pasciak. A preconditioning technique for indefinite systems resulting from mixed approximations of elliptic problems. Math. Comp., 50:1-17, 1988.

[2] J. H. Bramble and J. E. Pasciak. A domain decomposition technique for Stokes problems. Applied Num. Math., pages 251-261, 1989/1990.

[3] A. Brandt and N. Dinar. Multigrid solutions to elliptic flow problems. In S. V. Parter, editor, Numerical Methods for Partial Differential Equations, pages 53-147. Academic Press, New York, 1979.

[4] T. F. Chan and H. C. Elman. Fourier analysis of iterative methods for elliptic problems. SIAM Review, 31:20-49, 1989.

[5] H. Elman and D. Silvester. Fast nonsymmetric iterations and preconditioning for Navier-Stokes equations. SIAM J. Sci. Comput., 17:33-46, 1996.

[6] H. C. Elman. Perturbation of Eigenvalues of Preconditioned Navier-Stokes Operators. Technical Report UMIACS-TR-95-110, Institute for Advanced Computer Studies, University of Maryland, 1995. To appear in SIAM J. Matr. Anal. Appl.

[7] H. C. Elman and M. P. Chernesky. Ordering effects on relaxation methods applied to the discrete one-dimensional convection-diffusion equation. SIAM J. Numer. Anal., 30:1268-1290, 1993.

[8] H. C. Elman and G. H. Golub. Iterative methods for cyclically reduced non-self-adjoint linear systems. Math. Comp., 54:671-700, 1990.

[9] M. Fortin and R. Pierre. Stability analysis of discrete generalised Stokes problems. Numer. Meth. Partial Diff. Eq., 8:303-323, 1992.

[10] R. Freund and N. M. Nachtigal. QMR: a quasi-minimal residual method for non-Hermitian linear systems. Numer. Math., 60:315-339, 1991.

[11] G. H. Golub and A. J. Wathen. An Iteration for Indefinite Systems and its Application to the Navier-Stokes Equations. Technical Report AM-95-09, Mathematics Department, University of Bristol, 1995. To appear in SIAM J. Sci. Stat. Comput.

[12] P. M. Gresho, D. K. Gartling, J. R. Torczynski, K. A Cliffe, K. H. Winters, T. J. Garratt, A. Spence, and J. W. Goodrich. Is the steady viscous incompressible two-dimensional flow over a backward-facing step at Re=800 stable? Int. J. Numer. Meth. Fluids, 17:501-541, 1993.

[13] P. M. Gresho and R. L. Lee. Don't suppress the wiggles - they're telling you something. Computers and Fluids, 9:223-253, 1981.

[14] M. Gunzburger. Finite Element Methods for Viscous Incompressible Flows. Academic Press, San Diego, 1989.

[15] F. H. Harlow and J. E. Welch. Numerical calculation of time-dependent viscous incompressible flow of fluid with free surface. The Physics of Fluids, 8:2182-2189, 1965.

[16] R. A. Horn and C. R. Johnson. Topics in Matrix Analysis. Cambridge University Press, New York, 1991.

[17] O. A. Karakashian. On a Galerkin-Lagrange multiplier method for the stationary Navier-Stokes equations. SIAM. J. Numer. Anal., 19:909-923, 1982.

[18] T. A. Manteuffel and S. V. Parter. Preconditioning and boundary conditions. SIAM J. Numer. Anal., 27:656-694, 1990. 
[19] S. F. McCormick, editor. Multigrid Methods. SIAM, Philadelphia, 1987.

[20] M. F. Murphy and A. J. Wathen. On Preconditioners for the Oseen Equations. Technical Report AM 95-07, Department of Mathematics, University of Bristol, 1995.

[21] R. D. Richtmyer and K. W. Morton. Difference Methods for Initial Value Problems. John Wiley \& Sons, New York, second edition, 1967.

[22] T. Rusten and R. Winther. A preconditioned iterative method for saddle point problems. SIAM J. Matr. Anal. Appl., 13:887-904, 1992.

[23] Y. Saad. A flexible inner-outer preconditioned GMRES algorithm. SIAM J. Sci. Comput, 14:461-469, 1993.

[24] Y. Saad and M. H. Schultz. GMRES: A generalized minimal residual algorithm for solving nonsymmetric linear systems. SIAM J. Sci. Stat. Comput., 7:856-869, 1986.

[25] D. Silvester and A. Wathen. Fast iterative solution of stabilized Stokes systems part II: using block preconditioners. SIAM J. Numer. Anal., 31:1352-1367, 1994.

[26] R. Temam. Navier-Stokes Equations. North-Holland, Amsterdam, 1979.

[27] M. C. Thompson and J. H. Ferziger. An adaptive multigrid technique for the incompressible Navier-Stokes equations. J. Comput. Phys., 82:94-121, 1989.

[28] S. P. Vanka. Block-implicit multigrid solution of Navier-Stokes in primitive variables. J. Comput. Phys., 65:138-158, 1986.

[29] R. Verfürth. A combined conjugate gradient-multigrid algorithm for the numerical solution of the Stokes problem. IMA J. Numer. Anal., 4:441-455, 1984.

[30] G. Wittum. Multi-grid methods for the Stokes and Navier-Stokes equations. Numer. Math., $54: 543-564,1989$. 